\title{
Curva de aprendizaje del ingeniero en comunicaciones y electrónica del espacio aéreo
}

| Fecha de recibido: 18 de enero del 2021 | Fecha de aprobación: 10 de junio del 2021 |

\section{Rodolfo Martínez Gutiérrez \\ Doctor en Estudios del Desarrollo Global \\ Docente, Tecnológico Nacional de México , Campus Tijuana \\ México \\ Rol del investigador: escritura https://orcid.org/0000-0001-6501-9851 \\ $\triangle$ rodolfo.martinez@tectijuana.edu.mx}

\section{Carmen Esther Carey Raygoza}

Magíster en Gestión Ejecutiva Internaciona

Tecnológico Nacional de México, Campus Tijuana

Rol del investigador: escritura

https://orcid.org/0000-0002-3379-2273

$\bowtie$ carmen.carey@tectijuana.edu.mx

\section{Antonio Alfonso \\ Landero Mada}

Magíster en Administración

Tecnológico Nacional de México, Campus Tijuana

México

Rol del investigador: experimental https://orcid.org/0000-0002-2853-9783

$\bowtie$ antonio.landero19@tectijuana.edu.mx

\section{Carlos Hurtado Sánchez}

Doctor en Ciencias

Tecnológico Nacional de México, Campus Tijuana

México

Rol del investigador: escritura https://orcid.org/0000-0002-9913-592X $\triangle$ carlos.hurtado@tectijuana.edu.mx

Cómo citar este artículo: Martínez-Gutiérrez, R., Landeros-Mada, A. A., Carey-Raygoza, C. E., \& Hurtado-Sánchez, C. 2021.

Curva de aprendizaje del ingeniero en comunicaciones y electrónica del espacio aéreo. Ciencia y Poder Aéreo, 16(2), 128-147. https://doi.org/10.18667/cienciaypoderaereo.687 


\section{Curva de aprendizaje del ingeniero en comunicaciones y electrónica del espacio aéreo}

Resumen: Este estudio tiene como finalidad la investigación sobre el desarrollo de los ingenieros en comunicaciones y electrónica (ICE) que laboran en Servicios a la Navegación en el Espacio Aéreo Mexicano (SENEAM). Con el propósito de determinar cuáles han sido las transiciones y sus proyecciones en el entorno laboral durante su permanencia en dicho organismo, y conocer y desarrollar la curva de aprendizaje desde sus inicios profesionales como ICE en el área de Ingeniería de Servicios IDS, comunicaciones, radio ayudas o radar. Las curvas de aprendizaje son modelos empíricos que permiten estudiar las transformaciones tecnológicas como resultado del aprendizaje. Partiendo de esto, el aprendizaje es comprendido como la sabiduría adquirida por la repetición de un proceso (learning-by-doing) (Arrow, 1962). Wright (1996) publicó un artículo donde afirma que la curva de aprendizaje se observó por primera vez en 1920. Al respecto, Hirschmann (1964) comenta "que la práctica hace la perfección, que las cosas se pueden hacer mejor no solo la segunda vez, sino cada vez que lo intente"; la curva de aprendizaje es la que se encarga de cuantificar y representar gráficamente este rendimiento (Hirschmann, 1964). La SENEAM cuenta con más de 40 años en México; proporcionando servicios de ayuda a la navegación aérea para la seguridad, fluidez y orden en el espacio aéreo mexicano; garantizando calidad y eficiencia conforme a la normatividad nacional e internacional aplicable. Se espera que esta investigación dé a conocer los factores que determinan la curva de aprendizaje de los ingenieros en comunicaciones y electrónica, caso SENEAM.

Palabras clave: curva de aprendizaje; capacitación; mentoring.

Abstract: This work studied the progress made by the communications and electronics engineers (CEES) working at Servicios a la Navegación en el Espacio Aéreo Mexicano (SENEAM), with the aim of establishing their transitions and projections while working at this company, and acknowledging the learning curve by this staff from their beginnings as CEES in the corresponding area of IDS, communications, radio aids or radar operations. Learning curves are empirical models that allow studying technological transformations as a result of learning processes. From this, learning is understood as the knowledge we acquire from the repetition of a process (learning-by-doing) (Arrow, 1962). Wright (1936) published an article stating that the learning curve phenomenon was observed for the first time in 1920. On this regard, Hirschmann (1964) comments on the benefits of the learning curve that "practice makes perfect, and things can be done better not only the second time, but every time we try." Hence, the learning curve is the one in charge of quantifying and graphically representing this performance (Hirschmann, 1964). In this context, SENEAM, which has more than 40 years of experience in the Mexican aeronautical industry, provides air navigation assistance services with safety, fluidity and order, ensuring quality and efficiency in accordance with applicable national and international regulations. Consequently, this research is expected to reveal the factors that determine the learning curve of CEES in the airline industry through the case study of SENEAM.

Keywords: learning curve; training; mentoring.

Resumo: O objetivo deste estudo é a pesquisa sobre o desenvolvimento que vem sendo realizado com os engenheiros de comunicações e eletrônica (ICE) que trabalham nos Serviços de Navegação no Espaço Aéreo Mexicano (SENEAM). Isto a fim de determinar quais foram as transições e suas projeções no ambiente de trabalho durante sua gestão na referida organização, e conhecer e desenvolver a curva de aprendizado que tiveram desde o início profissional como ICE na área de IDS, comunicações, rádio ajudas ou radar. As curvas de aprendizagem são modelos empíricos que permitem estudar as transformações tecnológicas decorrentes da aprendizagem. Com base nisso, a aprendizagem é entendida como a sabedoria que adquirimos ao repetir um processo (learning-by-doing) (Arrow, 1962). Wright (1996) publicou um artigo afirmando que esse fenômeno da curva de aprendizado foi observado pela primeira vez em 1920. Sobre isso, Hirschmann (1964) comenta sobre os benefícios da curva de aprendizado "que a prática leva à perfeição, que as coisas podem ser feitas melhor não só na segunda vez, mas toda vez que você tentar"; a curva de aprendizado é a responsável por quantificar e representar graficamente esse desempenho (Hirschmann, 1964). Por outro lado, o SENEAM tem mais de 40 anos prestando serviços para a indústria aeronáutica no México; prestação de serviços de assistência à navegação aérea para segurança, fluidez e ordem no espaço aéreo mexicano; garantindo qualidade e eficiência de acordo com as regulamentações nacionais e internacionais aplicáveis.

Palavras-chave: curva de aprendizagem; treinamento; mentoria. 
La curva de aprendizaje es el resultado de la experiencia, del contacto del hombre con su entorno (Chango, 2014). La importancia de este trabajo está en conocer todas las funciones de un ingeniero en comunicaciones y electrónica (ICE) que labora en Servicios a la $\mathrm{Na}$ vegación en el Espacio Aéreo Mexicano (SENEAM).

SENEAM es un organismo desconcentrado de la Secretaría de Comunicaciones y Transportes (SCT) de México, el cual fue creado por decreto presidencial y publicado en el Diario Oficial de la Federación (DOF) del 3 de octubre de 1978 (DOF, 1978). Este organismo tiene como misión garantizar, a través de servicios a la navegación, el transporte seguro y eficiente de personas y bienes en el espacio aéreo mexicano. Para establecer la curva de aprendizaje hay que aportar información de la proyección para el desarrollo de las competencias sectoriales mediante la generación de un perfil de los ICE que laboran en el sector aeronáutico de México.

La importancia de una capacitación adecuada para el personal de ingeniería de servicios a las comunicaciones, radio ayudas y de radar de un organismo como SENEAM es primordial porque se deben de garantizar servicios a la navegación aérea con los más altos estándares y eficiencia, como lo marca la Organización de Aviación Civil Internacional (OACI) en el Anexo 10 y las regulaciones vigentes de la Agencia Federal de Aviación Civil en México.

En el paradigma para retroalimentar los conocimientos y las habilidades necesarias en los diferentes equipos y sistemas que se manejan en el sector aeronáutico, SENEAM cumple con las normas ISO 90012015, apartado 6.2 de recursos humanos que establece el sistema de gestión de la calidad.

Con la puesta en práctica, este estudio busca, mediante la curva de aprendizaje, que un ICE adquiera en un menor tiempo las competencias requeridas para conseguir un puesto en el sector aeronáutico, además de ser un recurso necesario para los administradores que requieren conocer cómo fue el desarrollo de la capacitación de los ICE que ingresan a laborar en el sector aéreo a través del tiempo. Al generar personal de ingeniería con una capacitación homogénea, continua y actualizada en los avances tecnológicos, se aporta en el desempeño óptimo de equipos de radio navegación y sistemas aeronáuticos utilizados en el sector aéreo.

\section{Surgimiento de la curva de aprendizaje}

En febrero de 1936, Patterson Wright público en Journal of the Aeronautical Sciences, volumen 3, el artículo titulado "Factores que afectan el costo de los aviones", la primera publicación que se tiene registrada sobre la curva de aprendizaje, la cual surgió de sus primeros estudios realizados en 1922 sobre la variación de los costos en el sector aeronáutico. En esta curva de aprendizaje, Wright representó la variación del trabajo empírico, y a partir de dos o tres puntos de la experiencia en la producción del mismo modelo en diferentes posibles cantidades a través de los años, esa curva, que al inicio mostró solamente la variación del trabajo, se utilizó para la estimación de propósitos y se fueron corrigiendo más datos hasta que quedó disponible y fue presentada en papel (Wright, 1936).

En su artículo de 1936, Wright afirma que el fenómeno de la curva de aprendizaje se observó por primera vez en 1920 en Dayton, Ohio, Estados Unidos, en la Fuerza Aérea Americana. Allí se encontró que en el ensamblaje de un segundo avión de cierto tipo se gastaba el $80 \%$ de las horas del primero. El octavo avión gastaba el $80 \%$ de las horas del cuarto, y así sucesivamente hasta llegar a un límite lógico (Chango, 2014).

\section{Hipótesis sobre la curva de aprendizaje}

La hipótesis de Wright era que las horas-hombre necesarias para completar una unidad de producción decrecerían en un porcentaje constante cada vez que la producción se duplicara (Chango, 2014). En la industria, la curva de aprendizaje es utilizada en el tiempo y al costo de la producción. En la figura 1 se puede 
observar en el eje $X$ la acumulación de lo que se ha aprendido, y en eje Y el tiempo invertido. Se conoce del surgimiento de la curva de aprendizaje en el sector aeronáutico desde Wright en 1920. Sin embargo, Terrazas et al. (2009) han indicado que la idea del aprendizaje individual y del aprendizaje organizacional se inició en la década de los setentas y lo aplicó Boston Consultan Group y de Conley.

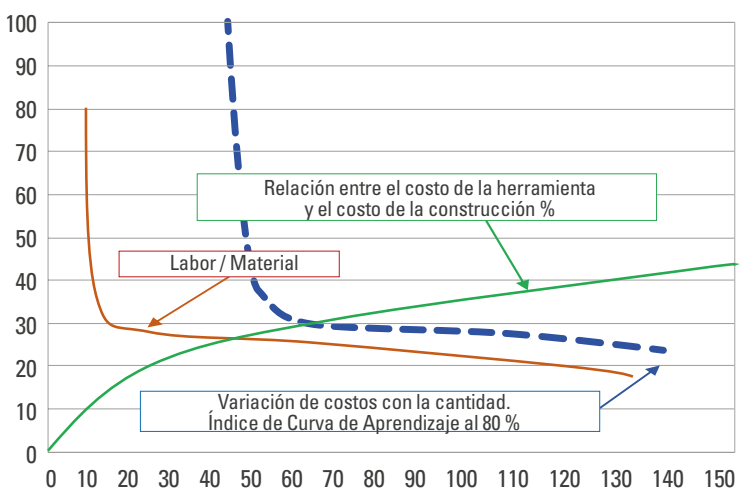

Figura 1. Curva de aprendizaje de 1936 Fuente: Wright (1936).

En la figura 2, la curva de aprendizaje muestra el trabajo. La forma general, la tendencia de los datos y la corrección de la curva se debieron a los nuevos puntos; estos correspondieron, a su vez, a los resultados de los datos de la experiencia adquirida, los cuales han permitido trazar otra curva que muestra la tasa de variación del material utilizado, el material comprado y el conjunto del avión contra la cantidad.

Por otra parte, Arrow (1962) propuso por primera vez la hipótesis sobre las implicaciones económicas del aprendizaje. Este modelo, uno de los más requeridos, plantea una tasa de aprendizaje que se describe como porcentaje, en la cual se disminuyen los costos una vez se duplica la capacidad de producción.

Las curvas de aprendizaje son modelos empíricos que facilitan el estudio de la evolución tecnológica como un resultado del conocimiento (Arrow, 1962). En este rubro, el aprendizaje se comprende como la experiencia que se adquiere por la repetición de un proceso (learning-by-doing); por ejemplo, los incrementos en la capacidad de producción dejan una experiencia debido a la repetición en un proceso productivo.

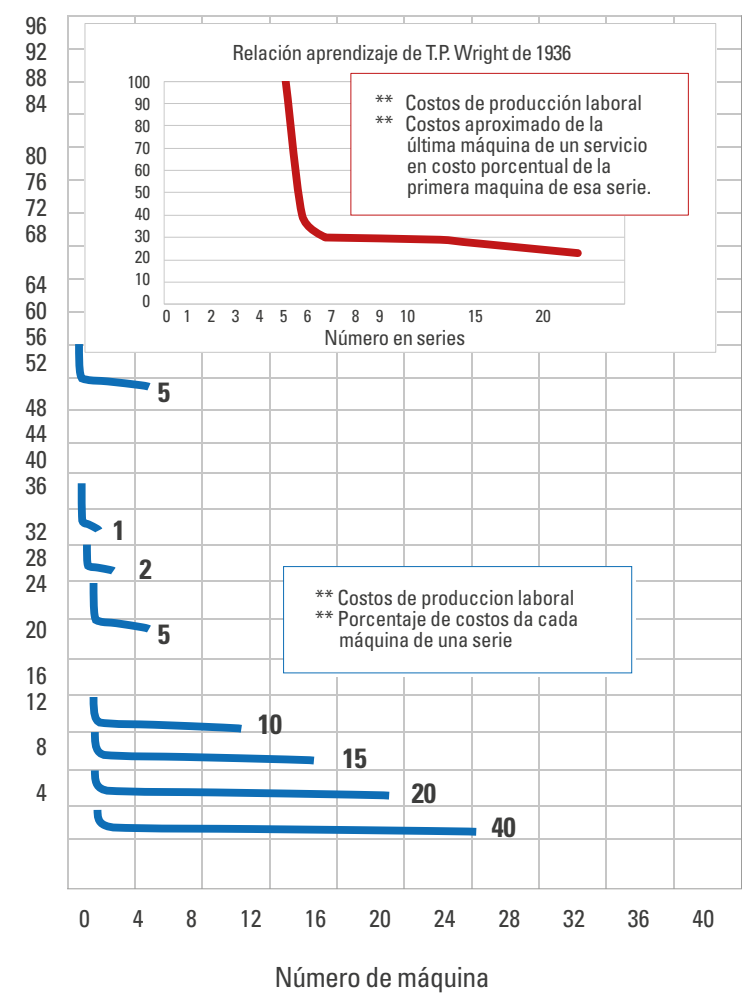

Figura 2. Curva de aprendizaje con la forma general y la tendencia (1936) Fuente: Wright (1936).

"La práctica hace la perfección", manifestó Hirschmann (1964). Siempre se puede hacer una cosa mejor, no solo la segunda vez, sino cada vez que se intente. Esto lo sabe todo el mundo, pero ¿cuántos saben que un patrón de mejora puede ser lo suficientemente regular como para ser predictivo? ¿Cuántos se dan cuenta de que tales patrones pueden caracterizar no solo el desempeño individual, sino también al desempeño compuesto de muchos individuos organizados para realizar una tarea común?

El rendimiento de la curva de aprendizaje es una característica natural, por lo tanto, se debe localizar dicho rendimiento no solo para más tipos de actividades ya registradas como receptivas, sino también para funciones poco probables, como aquellas que no se anunciaron previamente o que se creían susceptibles (Hirschmann, 1964). En la figura 3, los puntos muestran una tendencia decreciente para las horas de trabajo productivo en el mantenimiento y las paradas entre los años 1949 y 1956. Al final de ese periodo la trama parece nivelarse. 


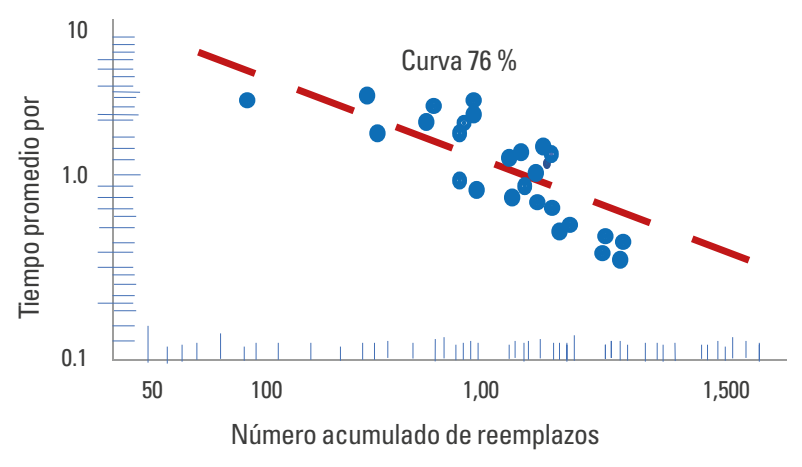

Figura 3. Curva de aprendizaje de mantenimiento en planta de General Electric

Fuente: Bennet (1957).

Si la administración hubiera especulado sobre esta curva, podría haber sentido que había alcanzado un nivel estable y que el mantenimiento había aprendido la mejor manera de hacer los trabajos requeridos para que no se produjera un nuevo descenso - al menos por un tiempo-. Pero, en realidad, la tendencia continuó, terminando en donde debería haberse esperado (Hirschmann, 1964). En ese sentido, el trabajo es mayor para los puestos que demandan de un alto grado de actividad manual y en los que, además, se están aplicando los métodos tradicionales. En ciertos casos es necesario mostrar al trabajador películas o videos en los cuales se presenten los procedimientos tradicionales y los movimientos nuevos que se estarán aplicando de la manera más efectiva, así como el hecho de que un hábito es una actividad que influye en el aumento de la productividad al reducir la necesidad de una reflexión consciente (Kanawaty, 1955). En la teoría de la mejora continua en una curva de aprendizaje se hace un esfuerzo continuo para mejorar el rendimiento del trabajador y que este sea más productivo (Willard \& Kantor, 1998).

Al realizar un análisis sobre el aprendizaje y su definición se detectan estos tres aspectos:

1. Es un cambio en la conducta o en cómo el individuo realiza sus labores porque asimiló una actividad y ya la hace de manera diferente a las anteriores veces.

2. Incluye el desenvolvimiento de una capacidad para manejarse. Esto quiere decir que la persona, conforme va conociendo las labores, va adquiriendo destrezas, desarrolla habilidades y adquiere competencias sectoriales que van a permanecer en el tiempo.

3. Es un resultado de la práctica donde va adquiriendo habilidades, destrezas y conocimientos que lo llevan a acumular una experiencia, por ejemplo, por el ensayo o error, o por observar a otros a través del ejemplo; algo similar ocurre cuando los seres humanos aprendemos a hablar.

\section{Funcionamiento de la curva de aprendizaje}

En el concepto de aprendizaje y con la intención de conocer el funcionamiento de las curvas de aprendizaje, que muestra los factores que influencian al aprendizaje. Estos pueden alterar de forma positiva o negativa su realización, entre estos factores se encuentran, por ejemplo, la velocidad del aprendizaje, la cual se mide en una relación del 80 \% y se la llama tasa de aprendizaje. Una gran cantidad de estas mejoras provienen de búsquedas que la gente realiza para perfeccionar el desempeño: se les conoce como mejoras en línea. Otras, por el contrario, provienen de distintas fuentes, entre las que se encuentran nuevas herramientas, nuevos materiales o reingenierías o mejoras fuera de línea. En la figura 4 se observan los elementos que han de marcar un aprendizaje como un proceso (Chango, 2014).

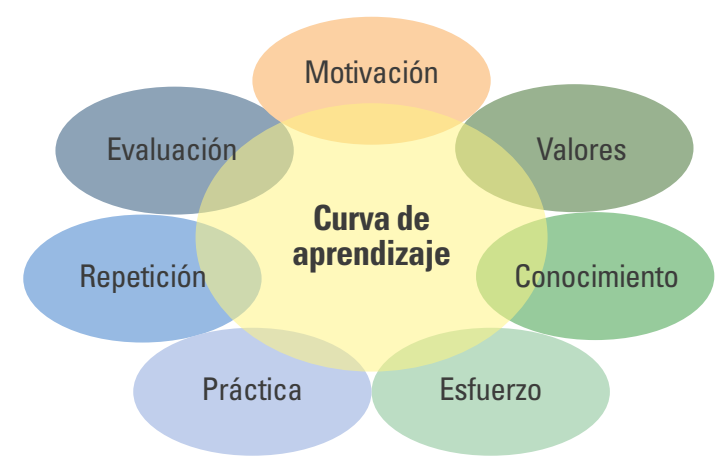

Figura 4. Elementos que influyen en el aprendizaje Fuente: Chango (2014). 
El aprendizaje se entiende como un proceso, y tiene elementos que lo demuestran: en cualquier proceso de aprendizaje existe una razón de necesidad y un interés intrínseco o extrínseco en la actividad; está conformado por valores comunes, información y datos; se motiva mediante el desafío, lo que evidencia un nivel de esfuerzo por lo esperado por el trabajador (Chango, 2014). La disciplina es una constante práctica y repetición que deja una experiencia en las tareas, en las que se acumulan conocimientos para reevaluar aprendizajes y propiciar una colaboración entre pares que sea relevante al momento de recibir la retroalimentación por una organización o por los involucrados.

\section{Método logarítmico de la curva de aprendizaje}

Diversos autores como Krajenski (2000) y Terrazas et al. (2009) indican que el método logarítmico va a facilitar la determinación de la curva de aprendizaje para cualquier unidad, TN, por la fórmula siguiente:

Método logarítmico $\quad T N=T O \times N b$

Donde:

$\mathrm{x}=$ Número de unidades.

$Y \mathrm{X}=$ Número de horas-hombre directas requeridas para producir la enésima $x$.

$\mathrm{K}=$ Número de horas-hombre directas requeridas para producir la primera unidad. $\mathrm{N}=\log$ va log2, donde $\mathrm{b}=$ porcentaje de aprendizaje.

\section{Estimación del porcentaje de aprendizaje}

Organizaciones y productos disponen de diferentes curvas de aprendizaje. La tasa de aprendizaje va a cambiar de acuerdo con la calidad de la gestión y del potencial de un proceso y del producto. En ese orden de ideas, la tasa de aprendizaje en el sector del espacio aéreo es del $85 \%$ (Chango, 2014). En los estudios realizados por Wright y que publicó en 1936 donde mencionaba que en un

\begin{abstract}
cuarto avión gastaba el $80 \%$ de las horas del segundo. El octavo avión gastaba el $80 \%$ de las horas del cuarto y así sucesivamente hasta llegar a un límite lógico. La velocidad del aprendizaje se mide con esta relación, (80 \%) y se llama tasa de aprendizaje. A menor tasa de aprendizaje, mayor al lapso de la curva de aprendizaje (esta es la única ocasión cuando $60 \%$ es mejor que $80 \%$ ) (Ballesteros et al., 2005, p. 185).
\end{abstract}

\section{Metodología}

Se realizó una investigación cualitativa con un tipo de muestreo por conveniencia no probabilístico, en el que se utiliza el razonamiento de una muestra para que esta sea más provechosa para el tema de investigación. Es un tipo de muestra intencional donde hay un informador clave que identifica a otro para entrevistar. Para efectos de una investigación integral, debe considerarse el enfoque sistémico y análisis del entorno sectorial (Martínez, 2012, 2020). De ese modo, se eligieron a 50 ingenieros con la condición de que laboraran en ingeniería de servicios en radio ayudas, comunicaciones y de radar en las diversas estaciones de la república mexicana. Del total, 47 fueron varones y 3 mujeres. En cuanto a los años de servicios que llevaban laborando en el organismo, el rango iba de los 5 hasta los 47 años.

\section{Diseño del instrumento}

Para la elaboración del diseño de la recopilación de información del instrumento de investigación se realizó una presentación de las variables de una forma confiable, válida y objetiva. Según los tratadistas, todo 
instrumento utilizado debe de reunir al menos dos condiciones: confiabilidad y validez (Rojas, 2011). Así, se utilizaron para la recopilación de la información los siguientes instrumentos:
a) Observación directa.
b) Cuestionarios.
c) Entrevistas.

Para la encuesta se usó la escala de Likert 5 con los valores: 5=excelente, 4=muy bueno, 3=bueno, 2=regular, 1=malo.

Para la validación de los instrumentos (encuestas y entrevista) se manejó el juicio de expertos (Skjong \& Wentworth, 2000). Como el juicio de expertos es conocida una opinión conocedora de individuos con recorrido en el tema, que son reconocidos por otros como peritos autorizados sobre determinado asunto, y que logran suministrar indagación, certidumbre, reflexiones y apreciaciones. El número de jueces que se debe manipular en un juicio depende del nivel de experticia y de la complejidad del discernimiento; la decisión sobre qué cantidad de expertos es la apropiada varía entre diferentes autores. Así, mientras Hyrkäs et al. (2003) sugieren un rango de 2 a 20 expertos, declara que 10 brindarían una evaluación confiable de la validez de contenido de un instrumento. Si un $80 \%$ de los peritos han estado de acuerdo con la validez de un ítem, este puede ser incorporado al instrumento. En esta investigación se compartió el instrumento con 15 expertos en los temas de capacitación y alto desarrollo en el organismo SENEAM, a quienes se les solicitó que evaluaran el instrumento de investigación, considerando sus apreciaciones y aportaciones para mejorar el instrumento.

En la tabla 1 se presenta un desglose de los factores que se miden en el cuestionario, así como los ítems de cada factor y el porcentaje que corresponde a cada uno de acuerdo con el total de los utilizados para la implementación de esta herramienta, donde se indica la variable a estudiar que aporta información para las conclusiones derivadas de la investigación.
Tabla 1

Desglose de los factores que se midieron en la muestra

\begin{tabular}{l|c|c|c|c}
\multicolumn{1}{c|}{ Factores } & Preguntas & $\begin{array}{c}\text { Total de } \\
\text { ítems }\end{array}$ & $\begin{array}{c}\text { Variable a } \\
\text { estudiar }\end{array}$ \\
\hline Información básica & $1-5$ & 5 & 3,42 & Independiente \\
\hline Desarrollo laboral & $6-8$ & 3 & 2,05 & Independiente \\
\hline Meritocracia & $9-11$ & 3 & 2,05 & Independiente \\
\hline SINCO & 12 & 16 & 10,96 & Independiente \\
\hline Funciones laborales & $13-15$ & 23 & 15,75 & Independiente \\
\hline Mentoring & $16-18$ & 3 & 2,05 & Independiente \\
\hline Competencias & $19-28$ & 10 & 6,85 & Independiente \\
\hline Capacitación & $29-42$ & 58 & 39,73 & Dependiente \\
\hline Curva de & $43-62$ & 20 & 13,7 & Dependiente \\
aprendizaje & & & & \\
\hline Satisfacción laboral & $63-64$ & 2 & 1,37 & Dependiente \\
\hline Motivación laboral & $65-66$ & 2 & 1,37 & Dependiente \\
\hline Equipos de trabajo & 67 & 1 & 0,68 & Dependiente \\
\hline Totales & & $\mathbf{1 4 6}$ & $\mathbf{1 0 0} \%$ & \\
\hline
\end{tabular}

Fuente: elaboración propia.

\section{Procedimiento}

Sobre la recolección de datos, Sampieri (2010) declara que es un proceso elemental, aunque el objetivo no es solamente determinar una variable para establecer conclusiones y estudios estadísticos; lo que se busca en una investigación cualitativa es conseguir datos (que se transforman en información) de individuos, poblaciones, problemáticas o cuestiones a profundidad. La recolección de datos se llevó a cabo en los entornos naturales y cotidianos de los encuestados 0 unidades de análisis. En el caso de las personas, en su entorno diario: cómo hablan, en qué creen, qué sienten, cómo piensan, cómo actúan.

Se utilizaron en la recolección de datos las herramientas tecnológicas disponibles, ya que los encuestados se encontraban en diversas localidades del territorio nacional en México.

En la recolección de datos se siguieron estos pasos:

1. Búsqueda de datos: en esta etapa se recolectó información que aportara conocimiento, mientras 
de forma paralela se llevó a cabo la recolección de los datos (donde un investigador fue instrumento). De igual manera, se analizaron los datos, transcribiendo la información que se iba recolectando a través de una bitácora de procesos en la que se registraban los resultados.

2. Recolección de datos: el instrumento del levantamiento de la información fue el mismo entrevistador, quien, a través de las herramientas aplicadas, recolectó los datos.

3. Se utilizaron las siguientes herramientas:
a) Encuesta.
b) Entrevistas.
c) Observación.

4. Análisis de datos: se inició con la conformación de todos los datos que se iban recabando y utilizando.
a) La ordenación de la información.
b) Registro del material.
c) Se utilizaron programas de cómputo para or- denar la información.

5. Análisis del material: los principales (y esenciales) métodos fueron el rigor, la validez y confiabilidad.
a) Dependencia.
b) Credibilidad.
c) Transferencia.
d) Confirmación.

6. Codificación de la información: se realizó la codificación de toda la información que se fue recibida a través de dos niveles:

a) Primer nivel: equiparación de los elementos para generar algunas clases.

b) Segundo nivel: equiparación de las siguientes categorías:

1. Análisis de datos.

2. Elaboración de patrones.

3. Origen de supuesto, explicaciones y las teorías.

Las etapas (actividades realizadas para llegar a las metas planteadas de la investigación y obtener una respuesta de los cuestionamientos del estudio), se juntaron porque eran repetitivas y persistentes; es decir, no hubo tiempos en el desarrollo donde se pudiera mencionar "aquí se concluye una etapa y sigue otra". Al ir observando en cada una y presenciar lo que ocurría, se analizaba y recolectaba información, y el análisis terminaba en actividades paralelas. Es decir, el análisis se iba desarrollando al mismo tiempo que se obtenía la información, de tal forma que la muestra inicial no era contundente si al continuar colectando la información evolucionaba el análisis.

\section{Aplicación del instrumento al personal de ingeniería de servicios (IDS)}

Rojas (2011) afirma que la validez es una cualidad del instrumento si este sirve para medir la variable que se busca medir y no otra; es decir, que sea el instrumento preciso y adecuado. Un instrumento de medición adecuado es aquel que registra datos observables que representan verdaderamente los conceptos o las variables que el investigador tiene en mente (Sampieri, 2010).

En esta investigación el investigador diseñó un cuestionario con preguntas dirigidas a recolectar información para cada una de las variables, así como la forma en que se administran para el registro de las observaciones que son propiedad del propio investigador del estudio, recolectando información dependiente de entrevistas a personal exitoso en su permanencia laboral en el organismo de distintas gerencias del país con el fin de obtener una muestra más precisa.

La información que se obtuvo de la aplicación del instrumento se analizó y clasificó para realizar una proyección del ICE en su vida laboral en el organismo SENEAM. Esta proyección está determinada por varios factores que aportan información utilizada para realizar la curva de aprendizaje del ICE del sector aéreo en SENEAM.

En la figura 5, se muestran los años laborados del personal de ingeniería que participó en la investigación. Se conoció el año de ingreso al organismo y, con 
eso, los años de servicio para la proyección en su carrera dentro del organismo. Se obtuvo, en primer lugar, que el $26 \%$ de los encuestados (13 personas) llevan "de 6 a 10 años" laborando en el organismo; en segundo lugar, que el $20 \%$ (10 personas) llevan laborando "de 31 a 35 años", y, en tercer lugar, que el $18 \%$ (9 personas) han trabajado "de 11 a 15 años".

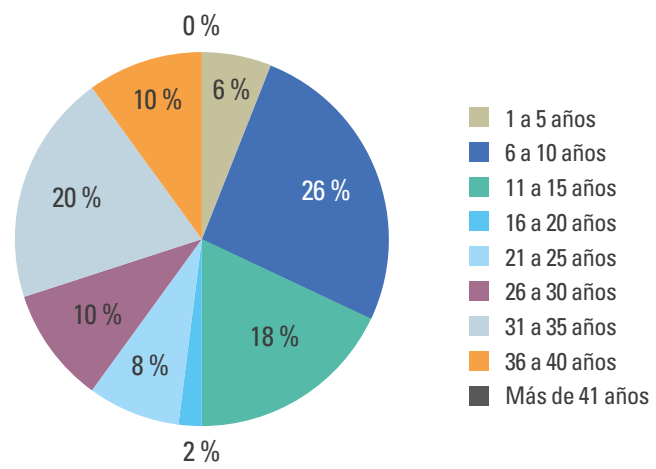

Figura 5. Años laborados en el organismo

Fuente: elaboración propia.

En la figura 6, área donde laboran los ingenieros que participaron en la muestra del estudio, se obtuvo que el $82 \%$ de la muestra, equivalente a 41 ingenieros, laboran en IDS comunicaciones y radio ayudas; que el $8 \%$ (4 encuestados) lo hacen en IDS Radar; otras 4 personas trabajan en Procesamiento radar, y el $2 \%$ (1 encuestado) trabaja en DISDA.

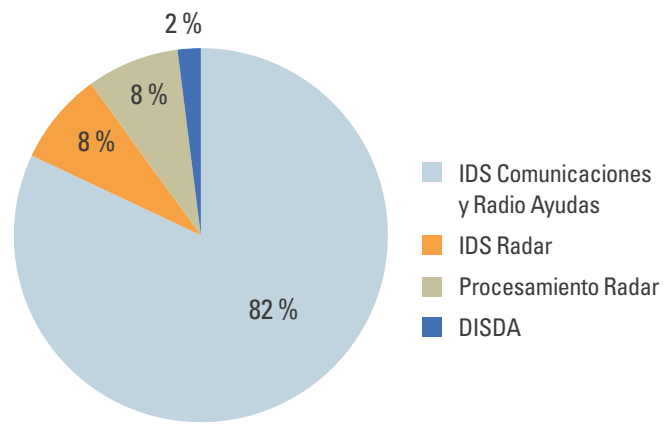

Figura 6. Área donde laboran los ingenieros que participaron en la muestra del estudio

Fuente: elaboración propia.

En la Figura 7, se muestra el tiempo transcurrido para que el ICE obtuviera su primera licencia aeronáutica como técnico aeronáutico clase II, emitida por la
AFAC, que lo habilita para intervenir equipos de radio ayudas o sistemas de radar. El $84 \%$ esperaron de 1 a 5 años en obtener su primera licencia aeronáutica; el $6 \%$ de 6 a 10 años, y otro $6 \%$ que manifestaron no tener licencia.

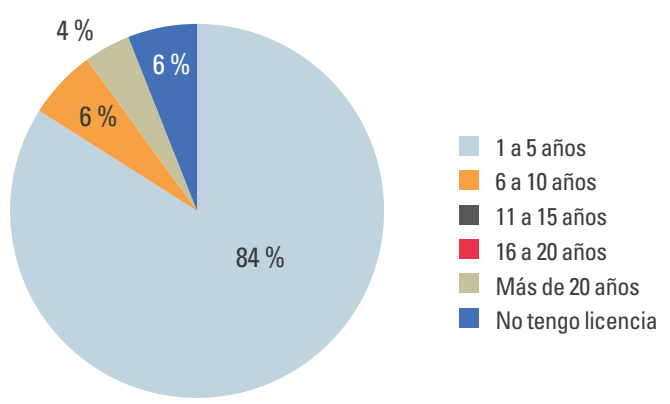

Figura 7. Tiempo transcurrido para obtener primera licencia aeronáutica Fuente: elaboración propia.

En la figura 8, se presenta el nivel con que ingresó el encuestado a laborar al organismo. En primer lugar, con el $88 \%, 44$ encuestados ingresaron con el nivel 69; en segundo y tercer lugar, el $4 \%$ ( 2 ingenieros) con el nivel 71 y con el nivel 73 , respectivamente, y, por último, el $2 \%$ (1 encuestado) con nivel 63.

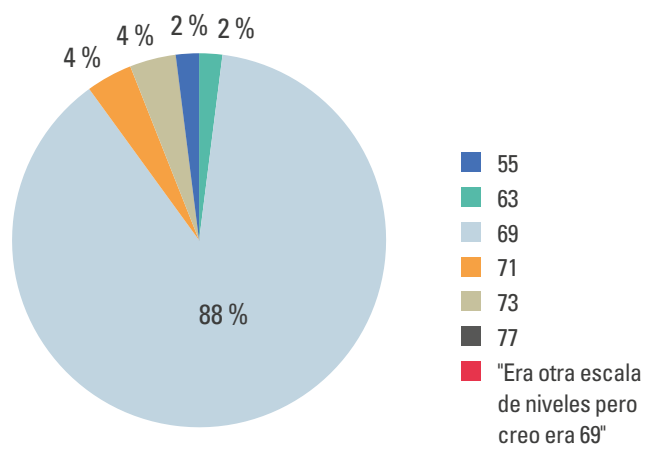

Figura 8. Nivel con que ingresó el encuestado a laborar al organismo Fuente: elaboración propia.

En la figura 9, se busca conocer los factores que influyen en el ascenso de nivel de los ICE. Al respecto, 45 ingenieros consideraron el comité de escalafón; 44 ingenieros señalaron a los sindicatos; 35 ingenieros indicaron la meritocracia; 20 ingenieros consideraron los méritos propios; 10 ingenieros eligieron el jefe inmediato, y 3 ingenieros respondieron "otros factores". 


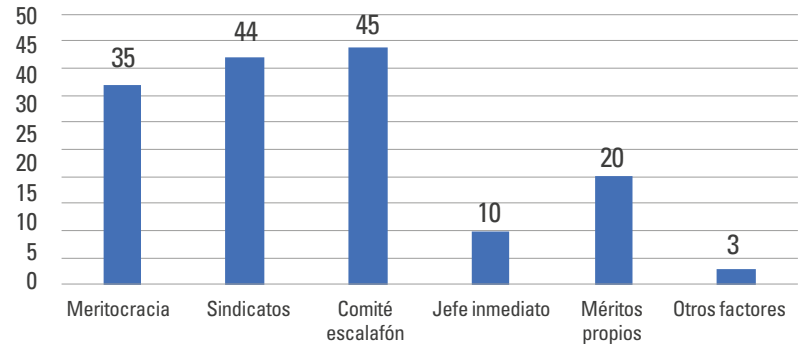

Figura 9. Factores que influyen en el ascenso de nivel de los ICE Fuente: elaboración propia.

El SINCO (Sistema Nacional de Clasificación de Ocupaciones) está conformado por un comité técnico de una serie de instituciones privadas y de gobierno de todos los sectores productivos de México, quienes aportan información de expertos y encuestas que, a través del Instituto Nacional de Estadística, Geografía e Informática, se llevan a cabo en todo el país (INEGI, 2011). El SINCO, en el grupo unitario 2281, menciona las 16 funciones laborales de los ingenieros en electrónica y telecomunicaciones. Estas funciones se presentan en la tabla 2, donde se evidencia que un $75 \%$ de los encuestados efectúan usualmente estas funciones.

Tabla 2

Funciones laborales de acuerdo con el SINCO

\begin{tabular}{l|c|c}
\multicolumn{1}{c|}{ Funciones laborales } & Cantidad & Porcentaje \\
\hline 1. Detectar y corregir fallas de equipo & 48 & $96 \%$ \\
\hline 2. Vigilar funcionamiento de equipo & 48 & $96 \%$ \\
\hline 3. Instalaciones de equipos y sistemas & 47 & $94 \%$ \\
\hline 4. Detectar y corregir fallas de sistemas & 46 & $92 \%$ \\
\hline 5. Elaborar diagramas técnicos por instalación & 40 & $80 \%$ \\
\hline 6. Dictar y/o aplicar normas técnicas para & 37 & $74 \%$ \\
\hline equipos de comunicaciones & 37 & $74 \%$ \\
\hline 7. Supervisar actividades de instalaciones & 36 & $72 \%$ \\
\hline 8. Elaborar diagramas para Tx y Rx de datos & 31 & $62 \%$ \\
\hline 9. Coordinar trabajos con proveedores externos & 30 & $60 \%$ \\
\hline 10. Definir procedimientos para instalaciones & 26 & $52 \%$ \\
\hline 11. Formular y aprobar cotizaciones & 25 & $50 \%$ \\
\hline 12. Redactar informes sobre diseños y proyectos & 18 & $36 \%$ \\
\hline 13. Diseñar proyectos para el desarrollo & 12 & $24 \%$ \\
\hline $\begin{array}{l}\text { 14. Normas para control y para el buen servicio } \\
\text { de los sistemas }\end{array}$ & $24 \%$ \\
\hline 15. Dirigir actividades de desarrollo de sistemas & 12 & $22 \%$ \\
\hline 16. Investigación para implementar sistemas & 11 & \\
\hline
\end{tabular}

Fuente: elaboración propia.
En la figura 10, se muestran las carreras de los encuestados. Todas las carreras cuentan con diferencias en cuanto a los perfiles requeridos por los planes de estudios diseñados por las universidades, es decir, que cuando los ingenieros sean contratados van a existir necesidades técnicas cognitivas que deberán ser cubiertas con una capacitación que proporcione una retroalimentación a los requerimientos que la industria del servicio en comunicaciones aeronáuticas requiere hoy en día.

En primer término, $62 \%$ (31 encuestados) estudiaron Ingeniería en Comunicaciones y Electrónica (ICE); en segundo lugar, el $18 \%$ (9 encuestados) son ingenieros en electrónica; en tercer lugar, con el $8 \%$, 4 encuestados son ingenieros en telecomunicaciones; el $4 \%$ ( 2 encuestados) estudiaron Ingeniería Médica; los demás encuestados, con el $2 \%$ cada uno (equivalente a 1 encuestado por carrera), estudiaron Ingeniería en Sistemas Computacionales, Ingeniería en Mecatrónica, Ingeniería Industrial en Electrónica y técnico electrónico.

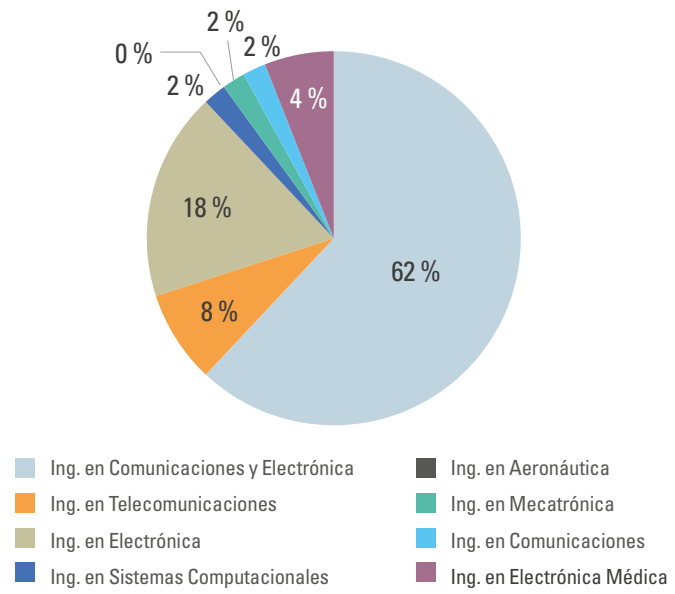

Figura 10. Carreras de estudio del encuestado

Fuente: elaboración propia.

La figura 11 aporta información sobre las competencias sectoriales; busca dar a conocer cómo los ingenieros consideran su capacitación y hacia donde está orientada para desarrollar las competencias sectoriales del personal de ingeniería. Se obtuvo que el $90 \%$ (45 ingenieros) respondieron que sí, y el $10 \%$ (con solo 5 ingenieros), que no. 


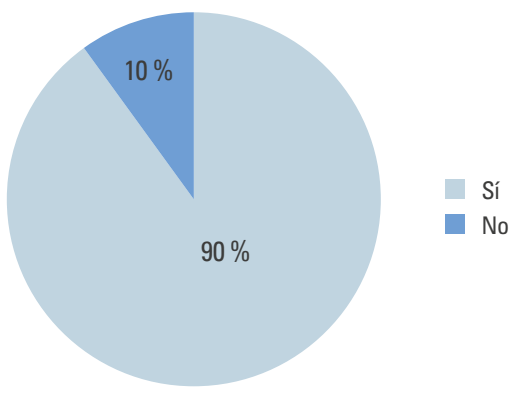

Figura 11. ¿Considera su capacitación orientada a desarrollar competencias sectoriales?

Fuente: elaboración propia

En la figura 12, se muestra el nivel meritocrático del ingeniero, el cual nos aporta una información sobre su proyección dentro del organismo, así como de las competencias sectoriales. En primer lugar, igualados con un $28 \%$ (14 encuestados), están los niveles 73 y 79; en segundo lugar, con $14 \%$ (7 ingenieros) el nivel 77; en tercer lugar, con el $12 \%$ (6 encuestados) el nivel 80.

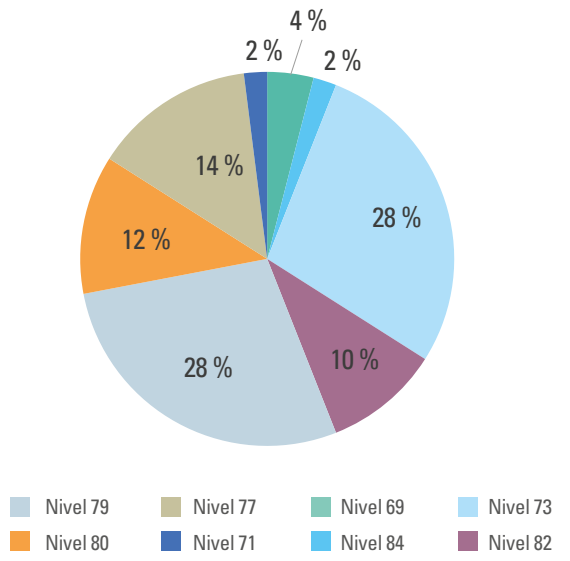

Figura 12. Nivel meritocrático

Fuente: elaboración propia.

En la figura 13, se aporta información sobre las competencias sectoriales, de donde se conoce si el ingeniero cuenta con una licencia aeronáutica emitida por la AFAC. El 82,1 \%, solamente 32 de los encuestados, contestaron que sí; mientras que el 17,9 \% (7 encuestados) contestaron que no tienen la licencia de técnico en aeronáutica tipo II que los habilita para radio ayudas o sistemas de radar.

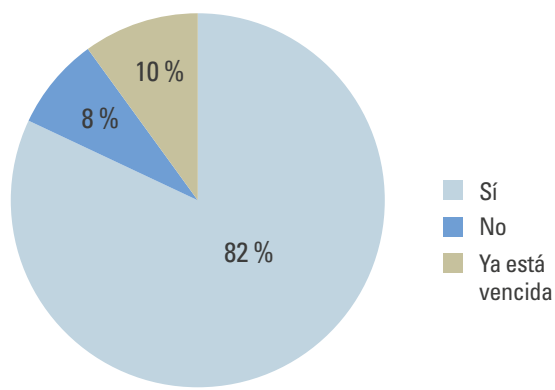

Figura 13. ¿Cuenta con una licencia aeronáutica? Fuente: elaboración propia.

La capacitación, según Dessler y Varela (2011), se refiere a los métodos que se utilizan para dar a los trabajadores nuevas habilidades que requieran para realizar sus labores de manera eficiente y con calidad.

En la figura 14, se muestra lo que se percibe en la capacitación inicial al recibir el curso de las materias básicas, que va a coadyuvar para mejorar los conocimientos cognitivos y sectoriales que existen en un ingeniero con diferente perfil en su formación académica, para que así se desempeñe con mejores fundamentos dentro del sector de las comunicaciones aeronáuticas. En primer término, con $46 \%$ cada cual, coincidieron 23 ingenieros por cada respuesta "sí la recibí" y "no la recibí". En segundo término, el 8 \% (4 ingenieros) mencionaron que no tuvieron curso inicial.

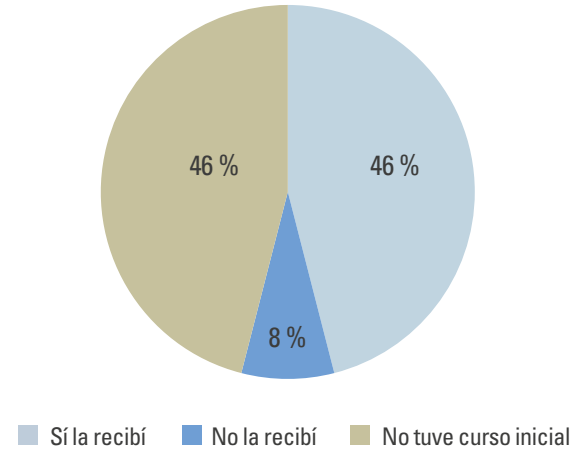

Figura 14 .¿Recibió curso de materias básicas en su capacitación inicial? Fuente: elaboración propia

En la tabla 3, se exhiben los cursos de capacitación para los ingenieros que laboran en ingeniería de servicios en comunicaciones, radio ayudas y radar, 
específicamente, el tiempo de duración de sus cursos de capacitación para diferentes equipos y sistemas aeronáuticos con el porcentaje de quienes los han recibido.

Tabla 3

Cursos de capacitación para IDS comunicaciones en lo general

\begin{tabular}{l|c|c|c}
\hline \multicolumn{1}{c|}{ Sistema } & Duración (h) & Cantidad & Porcentaje \\
\hline 1. VOR & 280 & 20 & $40 \%$ \\
\hline 2. DME & 140 & 20 & $20 \%$ \\
\hline 3. VCS & 35 & 35 & $70 \%$ \\
\hline 4. ILS & 420 & 3 & $6 \%$ \\
\hline 5. Transmisión de datos & 35 & 43 & $86 \%$ \\
\hline 6. Sistemas de modulación & 35 & 42 & $84 \%$ \\
\hline 7. Antenas & 35 & 40 & $80 \%$ \\
\hline 8. Comunicaciones & 35 & 39 & $78 \%$ \\
\hline 9. Telefonía & 35 & 35 & $6 \%$ \\
\hline 10. Radar & 210 & 10 & $20 \%$ \\
\hline 11. AFTN & 35 & 33 & $66 \%$ \\
\hline 12. VCS & 35 & 35 & $70 \%$ \\
\hline 13. VCX & 35 & 28 & $56 \%$ \\
\hline 14. DIVOS & 35 & 33 & $66 \%$ \\
\hline 15. Transmisores y receptores & 35 & 32 & $64 \%$ \\
\hline 16. NDB & 35 & 2 & $4 \%$ \\
\hline 17. Equipos meteorológicos & 35 & 30 & $60 \%$ \\
\hline 18. Comunicación satelital & 35 & 32 & $64 \%$ \\
\hline 19. Sistemas de energía & 35 & 44 & $88 \%$ \\
\hline 20. Redes de datos & 35 & 22 & $44 \%$ \\
\hline 21. Capacitación de sistemas & 35 & 16 & $32 \%$ \\
\hline 22. Anexo 10 & 35 & 44 & $88 \%$ \\
\hline 23. ATIS & 35 & 10 & $20 \%$ \\
\hline 24. WAAS & 35 & 1 & $2 \%$ \\
\hline 25. LEITCH & 35 & 8 & $16 \%$ \\
\hline & & & \\
\hline
\end{tabular}

Fuente: elaboración propia.

\section{Conclusiones}

Con base en los resultados obtenidos se desarrolló la curva de aprendizaje de los ingenieros en comunicaciones y electrónica que laboran en el sector aéreo en SENEAM.

El conocimiento se deprecia si no existe la innovación y la tecnología. Debido a la falta de presupuestos o de una planeación estratégica adecuada, según Jacobs (2014), estos podrían ir disminuyendo, de ahí la importancia de mantenerse a la vanguardia en los ámbitos tecnológicos. Se consideró un porcentaje de aprendizaje según la industria. De acuerdo con Jacobs (2014), el porcentaje de índice de aprendizaje para el sector aeronáutico del espacio aéreo es del $85 \%$.

Existen diferencias entre el índice de aprendizaje de una organización como SENEAM en el ramo de los servicios de radio comunicaciones aeronáuticas y de los servicios del tránsito aéreo y otras industrias que por convención manejan otro índice de aprendizaje. Dichas diferencias ocurren debido a las diversas características de las operaciones derivadas de los equipamientos, los métodos de trabajo, lo servicios que ofrecen, la organización de su planta productiva y por las diferencias de los procedimientos que se manifiestan en el desarrollo del porcentaje del aprendizaje mismo.

En esta investigación el sujeto de estudio fue el personal del área de ingeniería que cuenta con la carrera de Ingeniería en Comunicaciones y Electrónica (ICE) o carreras afines. De acuerdo con Lefcovich (2003), una curva de aprendizaje es la elaboración de un censo gráfico de los progresos que se dan en los costos mientras el organismo gana experiencia y sube el número total de artefactos que se producen en las líneas de producción o de ensamble. Para conocer las respuestas a las variables, en esta investigación se hizo un censo de los progresos en las capacitaciones en las cuales los ingenieros, por medio de sus cursos, han acumulado horas de experiencia. El censo se hizo a través de herramientas de recolección de la información como la encuesta y la entrevista. De esa manera, el organismo ha ganado con la curva de la experiencia o curva de aprendizaje de su capital humano y, además, en competencias sectoriales.

\section{Determinar la curva de aprendizaje de las funciones de los ICE del sector aéreo}

a) Inicialmente, se conoció en qué área laboran los encuestados, los años de servicio para su proyección y el porcentaje de conocimiento sobre 
las funciones laborales que determina el SINCO en el grupo unitario 2281 que realizan los ICE encuestados. El $80 \%$ de los cuarenta encuestados contestó que "sí, muy bien", y el $20 \%$ contestó "sí, bien".

b) Se rectificaron las funciones que intervienen para la determinación de la curva de aprendizaje en los ICE del sector aéreo caso SENEAM. Para esto, se describieron las actividades que se necesitan para desarrollar la curva de aprendizaje sobre los tiempos de capacitación de los equipos y sistemas en los cuales los ICE desarrollan sus funciones, así como la "n" cantidad de cursos que han tomado en su formación dentro del organismo.

c) Con respecto a las causas del problema, y después de un esbozo del planteamiento de la investigación, se establecieron los siguientes factores que determinan la curva de aprendizaje de los ICE del sector aéreo:

1. Desarrollo laboral.

2. Capacitación.

3. Funciones laborales del ICE.

4. La meritocracia en la administración pública.

5. Sistema Nacional de Clasificación de Ocupaciones (SINCO).

6. Satisfacción laboral.

7. Motivación laboral.

8. Equipos de trabajo.

9. Mentoring.

10. Competencias sectoriales.

d) Para la realización de la curva de aprendizaje se utilizaron los datos de los diversos sistemas que son utilizados por los ICE del sector aéreo en SENEAM.

e) Se utilizó el método logarítmico para el cálculo de la curva de aprendizaje.

f) La recaudación de la información para obtener los datos necesarios y realizar los cálculos se hizo por medio de encuestas con los ICE.

g) Se realizó el cálculo de la fórmula del método logarítmico. Diversos autores como Krajenski (2000), Terrazas et al. (2009) y Jacobs (2014) indican que el método logarítmico va a facilitar la determinación de la mano de obra para cualquier unidad.
Ya conocidos todos los datos, se procedió a realizar el modelo de la curva de aprendizaje teniendo en consideración la tasa del índice de aprendizaje que, según Jacobs (2014), es del 85 \% en el sector aéreo. Así, se realizó y se proyectó la curva de aprendizaje para cada uno de los niveles $(69,71,73,77,79,80,81,82)$ que existen en las categorías laborales de los ICE dentro del organismo. Cabe resaltar que la curva es aplicable tanto de forma individual como organizacional

Por otra parte, el aprendizaje que se adquiere de forma individual es el mejor resultado que se esperaría de los ingenieros que están recibiendo una capacitación constante debido a que este proceso les dará las habilidades y la eficiencia en virtud de su propia experiencia.

En el ámbito del aprendizaje organizacional existen diferentes tipos de capacitación, pero todas, al final, conforman una sola curva de aprendizaje, en donde el conocimiento que se está acumulando durante todo un periodo se transforma en un capital de conocimientos intangible para la organización, pero que aporta enormemente para la curva de aprendizaje del organismo. En todo este proceso de la curva de experiencia se adquieren conocimientos, experiencias, habilidades y destrezas, es decir, "la práctica hace al maestro".

La teoría de la curva del aprendizaje se basa en tres suposiciones (Chango, 2014), las cuales se comprueban en esta investigación:

1. La cantidad de tiempo requerido para completar una tarea o unidad determinadas disminuirá cada vez que se repita la tarea.

2. La unidad de tiempo disminuirá en una razón decreciente.

3. La reducción del tiempo seguirá un patrón fijo.

En la tabla 4, se realiza una proyección de la curva de aprendizaje a través de la capacitación de los ICE en SENEAM, de acuerdo con los datos recabados tanto en las encuestas como en las entrevistas a personal de IDS, que han sido personal con casos de éxito. Además, se tomaron muestras de todas las regiones del país que componen el organismo. Esta tabla de proyección se utilizó para la realización de la curva de aprendizaje. 
Tabla 4

Proyección de capacitación de un ICE en SENEAM

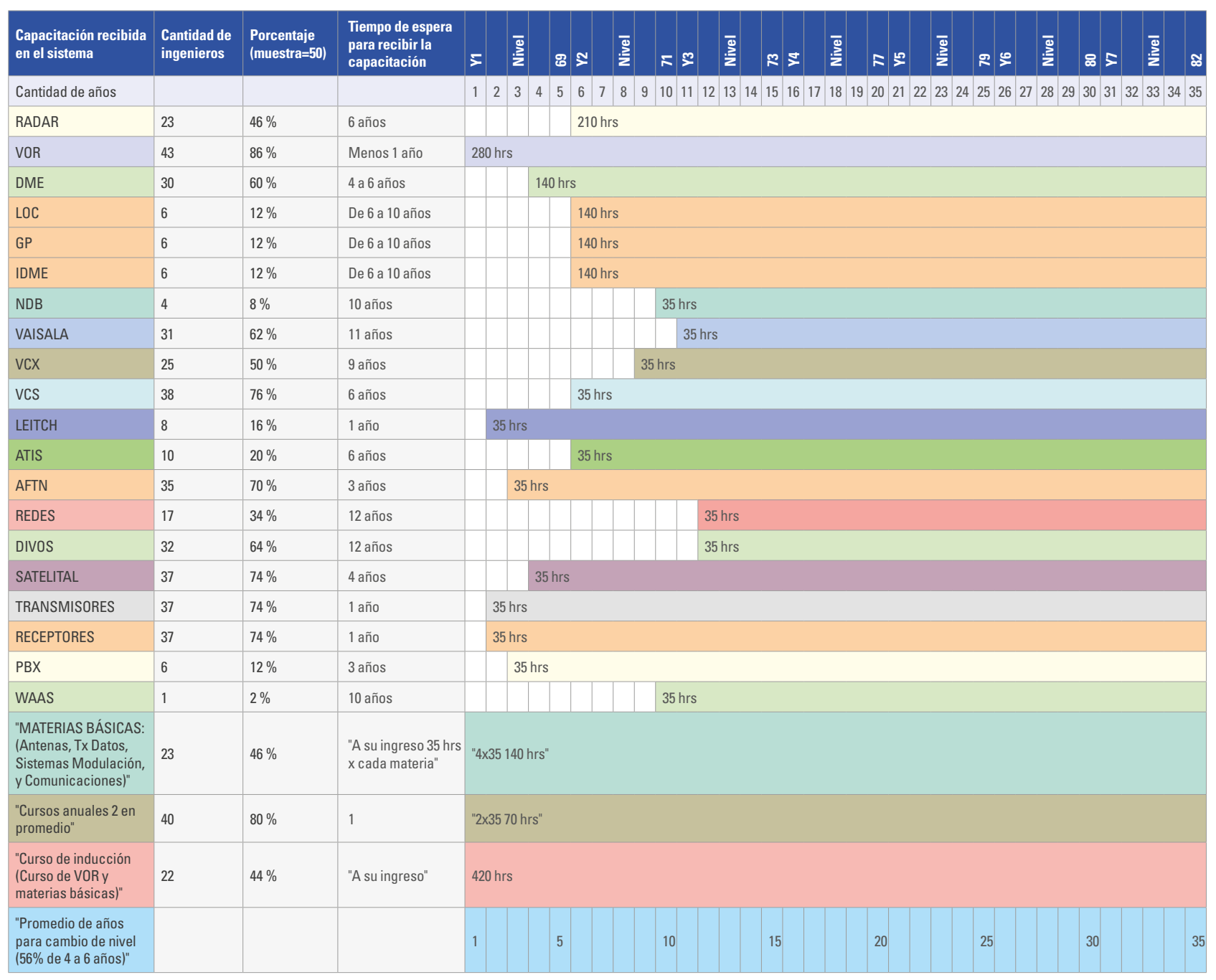

Fuente: elaboración propia.

En la tabla 5, se presentan los datos para el cálculo de la curva de aprendizaje de los ICE con el nivel 69 mediante el método logarítmico. Este es un nivel de inicio para un ingeniero de reciente ingreso al organismo dentro del área de IDS comunicaciones, radar y radio ayudas. Los datos de la columna K=Horas y de $\mathrm{N}=\mathrm{Cur}-$ sos, se obtuvieron de la tabla 4 sobre la proyección de capacitación del ICE en SENEAM. Así sucede con todos los cálculos de las curvas de aprendizaje en todos los niveles. De la tabla 4 de proyección se obtiene que va de acuerdo con la meritocracia y a la capacitación de cada nivel del ICE.
Tabla 5

Datos de cálculo de curva de aprendizaje para el nivel 69

\begin{tabular}{c|c|c|c|c|c|c}
$\begin{array}{c}\mathbf{X}= \\
\text { Cursos }\end{array}$ & $\begin{array}{c}\mathbf{K}= \\
\text { Horas }\end{array}$ & $\log \mathbf{B}$ & $\log \mathbf{2}$ & $\log \mathbf{B} / \log \mathbf{2}$ & $\begin{array}{c}\mathbf{X E l e v a d o} \\
\mathbf{a} \mathbf{l} \mathbf{~ N}\end{array}$ & $\mathbf{Y}$ \\
\hline 1 & 420 & $-0,070581074$ & 0,301029996 & $-0,234465254$ & 1 & 420 \\
\hline 2 & 140 & $-0,070581074$ & 0,301029996 & $-0,234465254$ & 0,85 & 119 \\
\hline 3 & 70 & $-0,070581074$ & 0,301029996 & $-0,234465254$ & 0,772914837 & 54,10403857 \\
\hline 4 & 35 & $-0,070581074$ & 0,301029996 & $-0,234465254$ & 0,7225 & 25,2875 \\
\hline 5 & 35 & $-0,070581074$ & 0,301029996 & $-0,234465254$ & 0,685671062 & 23,99848715 \\
\hline 6 & 35 & $-0,070581074$ & 0,301029996 & $-0,234465254$ & 0,656977611 & 22,99421639 \\
\hline 7 & 35 & $-0,070581074$ & 0,301029996 & $-0,234465254$ & 0,63365647 & 22,17797645 \\
\hline 8 & 35 & $-0,070581074$ & 0,301029996 & $-0,234465254$ & 0,614125 & 21,494375 \\
\hline 9 & 35 & $-0,070581074$ & 0,301029996 & $-0,234465254$ & 0,597397345 & 20,90890707 \\
\hline
\end{tabular}

Fuente: elaboración propia. 
En la figura 15, se presenta la curva de aprendizaje del nivel 69, donde se van acumulando cursos de capacitación durante sus inicios en el organismo dentro de los 4 a 6 años.

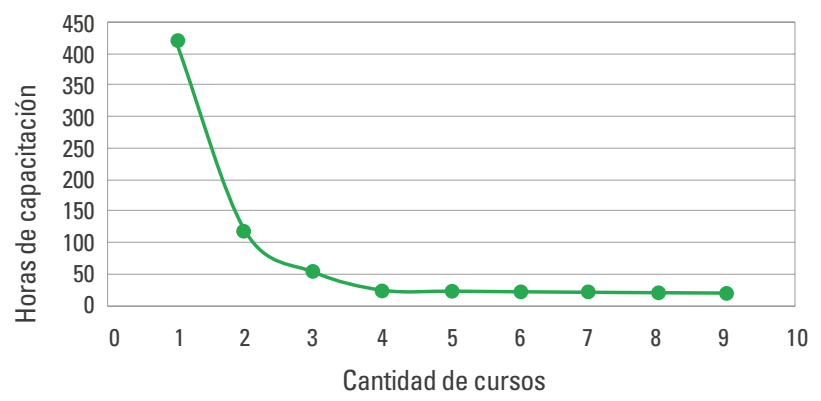

Figura 15. Curva de aprendizaje del nivel 69 Fuente: elaboración propia.

Se observa que la curva desciende de izquierda a derecha e indica que la experiencia hace descender los costos a medida que aumenta la producción o el aprendizaje. Los costos van a ir disminuyendo más lentamente que la experiencia acumulada; esto significa que a medida que pasa tiempo y se madura en la experiencia de los equipos y sistemas se vuelve más difícil la reducción de los costos, debido a que poco a poco se van reduciendo los gastos en la capacitación por la experiencia que se adquiere al momento de una capacitación adecuada al ingreso de un ICE al organismo.

En el eje de las abscisas (eje Y) se mide el número acumulado del tiempo invertido en la capacitación del ICE durante el ingreso a laborar al organismo. En el eje de las ordenadas (eje X) se mide el número de cursos de capacitación que se necesitan para lograr la curva de aprendizaje. Se concluye que la curva de aprendizaje es atípica, ya que ninguna curva es del todo uniforme, pues siempre existen las fluctuaciones en su inclinación y demás factores que influyen en su determinación (Yelle, 1979; Titone, 1986; Kelly, 1982).

Se comprobó la curva de aprendizaje tal y como fue planteada por Wright (1936). En ella se observa una pendiente empinada que indica un aprendizaje duro y difícil, mientras que a medida que se va haciendo plana indica un aprendizaje fácil y eficiente; en el eje de las $X$ se observa la acumulación de lo aprendido y en el eje de las $Y$ el tiempo invertido.

\section{Perfil del ingeniero en comunicaciones y electrónica del sector aéreo de México}

Producto de la investigación realizada, se obtuvo un perfil del ingeniero en comunicaciones y electrónica que labora en el sector aeronáutico de México. Se encontraron sus competencias y cómo la meritocracia influye en el desarrollo de las mismas. La determinación de la curva de aprendizaje permitió conocer el perfil del ICE del sector aéreo, que se propondrá para ser utilizado por el SINCO en el 2021 en el catálogo de puestos, con la descripción y funciones que realizan los ingenieros del sector aéreo de México. Asimismo, se elaboró un perfil que incluye los conocimientos, los valores y las destrezas que están relacionadas con los saberes básicos que se tienen al inicio de la capacitación del ingeniero, y que son necesarios dentro del sector aeronáutico si se tienen en cuenta las condiciones de trabajo para la solución de los problemas detectados en los sistemas y equipos aeronáuticos (véase tabla 6).

Tabla 6

Conocimientos para el perfil del ICE del sector aeronáutico

\begin{tabular}{|c|c|c|c|}
\hline $\begin{array}{l}\text { Ciencias } \\
\text { básicas }\end{array}$ & $\begin{array}{c}\text { Conocimientos } \\
\text { generales }\end{array}$ & $\begin{array}{l}\text { Conocimientos } \\
\text { de ingeniería }\end{array}$ & $\begin{array}{l}\text { Destrezas } \\
\text { y valores }\end{array}$ \\
\hline Matemáticas & Administración & Circuitos eléctricos & $\begin{array}{l}\text { Toma de } \\
\text { decisiones }\end{array}$ \\
\hline Física & Inglés & $\begin{array}{l}\text { Procesamiento de } \\
\text { señales (análogas } \\
\text { - digitales) }\end{array}$ & $\begin{array}{l}\text { Capacidad } \\
\text { de análisis }\end{array}$ \\
\hline Programación & $\begin{array}{l}\text { Gestión de } \\
\text { recursos }\end{array}$ & Electrónica analógica & $\begin{array}{l}\text { Ética } \\
\text { profesional }\end{array}$ \\
\hline $\begin{array}{l}\text { Teoría } \\
\text { Electromagnética }\end{array}$ & $\begin{array}{l}\text { Sistemas } \\
\text { informáticos }\end{array}$ & Electrónica digital & Responsabilidad \\
\hline \multirow[t]{11}{*}{$\begin{array}{l}\text { Antenas y } \\
\text { propagación }\end{array}$} & $\begin{array}{l}\text { Equipos de } \\
\text { medición }\end{array}$ & $\begin{array}{l}\text { Sistemas de modulación } \\
\text { AM-FM }\end{array}$ & Trabajo en equipo \\
\hline & & Radio ayudas & \\
\hline & & $\begin{array}{l}\text { Comunicaciones } \\
\text { tierra-aire }\end{array}$ & \\
\hline & & Sistemas de energía & \\
\hline & & $\begin{array}{l}\text { Redes de comunicación } \\
\text { de datos }\end{array}$ & \\
\hline & & Telefonía digital & \\
\hline & & $\begin{array}{l}\text { Comunicaciones } \\
\text { satelitales }\end{array}$ & \\
\hline & & Sistemas de microondas & \\
\hline & & $\begin{array}{l}\text { Comunicaciones } \\
\text { inalámbricas }\end{array}$ & \\
\hline & & Redes aeronáuticas & \\
\hline & & Redes TCP-IP & \\
\hline
\end{tabular}

Fuente: elaboración propia. 
Se desarrollan competencias sectoriales de acuerdo con la capacitación que se está recibiendo mientras se asimilan los cambios tecnológicos que existen de manera acelerada en la industria aeronáutica.

\section{De acuerdo con las ciencias básicas}

1. Conocimientos y habilidades matemáticas para la realización de diferentes cálculos necesarios en las mediciones eléctricas.

2. Conocimientos de física para la realización de diferentes cálculos relacionados con el uso de vectores utilizados en la capacitación de equipos de radio ayudas.

3. Conocimientos de programación para el diseño de sistemas y de interfaces electrónicos.

4. Conocimientos en la teoría electromagnética para la comprensión de los fenómenos eléctricos y magnéticos que se utilizan en la electrónica y comunicaciones.

\section{De acuerdo con los conocimientos generales}

5. Conocimientos de administración para mantener en orden y bajo control las actividades que se desarrollan.

6. Conocimientos de un idioma extranjero como el inglés, pues es el idioma mundial de la aeronáutica y se requiere para capacitación, coordinaciones, pruebas y documentación de equipos y sistemas.

7. Conocimientos sobre antenas y propagación. Las antenas son requeridas para recibir y emitir señales electromagnéticas; al transmitir o recibir, propagan señales electromagnéticas a través de diversos medios.

8. Conocimientos para la gestión de recursos tanto financieros como humanos para llevar a cabo los diversos proyectos.

9. Conocimientos en sistemas informáticos para el manejo de los diversos software y hardware que se utilizan en la industria aérea.

10. Tener la habilidad de trabajar en equipo ayuda para compartir conocimientos y estimula el aprendizaje individual y de un grupo.
11. Conocimientos en circuitos eléctricos para el conocimiento del transporte de la energía eléctrica a través de los cableados y dispositivos electrónicos.

12. Conocimientos de procesamientos de señales analógicas y digitales para conocer una manipulación matemática de una señal de información, para cambiarla o mejorarse en algún sentido, y se utiliza en diversos equipos de procesamiento de voz y comunicaciones.

13. Conocimientos de electrónica analógica para el manejo de los voltajes, corrientes, resistencias, impedancias, potencia.

14. Conocimientos de electrónica digital para los componentes discretos utilizados en los equipos electrónicos.

15. Conocimientos de sistemas de modulación en AM y FM para el uso en las comunicaciones radio eléctricas en diversos equipos y sistemas aeronáuticos.

16. Conocimientos en radio ayudas para equipos como el Localizador, GP o Trayectoria de Planeo, IDME, VOR, DME, NDB.

17. Conocimientos en las comunicaciones tierra-aire, que se efectúan a través de equipos de radio en frecuencias aeronáuticas y son necesarias para la comunicación entre los controladores de tránsito aéreo y los pilotos de las aeronaves, así como de los controladores de torre con personal terrestre.

18. Conocimientos en sistemas de energía eléctrica para el suministro de la energía que brinda el funcionamiento de los equipos aeronáuticos y de comunicaciones.

19. Conocimientos en redes de comunicaciones de datos para el envío y recepción de la información a través de diversos medios como fibra óptica, terrestre, antenas y enlaces por protocolo de internet.

20. Conocimientos de telefonía digital para las grabadoras de voz y enlaces de voz.

21. Conocimientos en comunicaciones satelitales para los enlaces de voz y datos que se utilizan en una red nacional. 
22. Conocimientos en sistemas de microondas utilizados en la transmisión de voz y enlaces de datos para redes o monitoreos de equipos aeronáuticos.

23. Conocimientos de enlaces inalámbricos, los cuales facilitan la operación en lugares donde las computadoras u otros dispositivos no se encuentran en una ubicación fija.

24. Conocimientos de redes aeronáuticas para el intercambio de información en la red aeronáutica mundial como la AFTN.

25. Conocimientos en redes TCP-IP, las cuales se utilizan en la operación proporcionando comunicación de las computadoras con servidores o la internet.

\section{De acuerdo con las destrezas y valores}

26. Habilidades en la toma de decisiones para realizar una elección entre las opciones o formas para resolver situaciones que se presenten.

27. Habilidad para realizar análisis y poder procesar la información que ayude a tomar las mejores decisiones para obtener buenos resultados.

28. Mantener la ética profesional es importante para conducirse de acuerdo con las normas y valores que rigen el actuar de un trabajador en la organización y para trabajar en conjunto por el bien común.

29. Mantener la responsabilidad para contar con un nivel de compromiso que se asume por el personal para lograr una mejor posición en el organismo.

30. Mantener el trabajo en equipo se requiere en las diversas labores para compartir actividades en común que se designan, de tal manera que se cuide la seguridad de los integrantes del equipo y se logre el objetivo asignado.

\section{Elementos que influyen en la conformación de una curva de aprendizaje}
a) La edad.
b) Conocimientos de las funciones del trabajo a de- sarrollar.

c) Empatía para aprender.

d) Capacidad de concentración.

e) Talento del individuo.

f) Diseño de procesos.

g) Métodos de mejora continua o kaizen.

h) Materiales o herramientas de trabajo.

\section{Riesgos de ser indiferente ante la curva de aprendizaje}

En una organización la curva de aprendizaje es considerada primordial para el desarrollo y la capacitación del personal de ingeniería, debido a los riesgos que existen propios de las funciones de los ICE (Acá, 2017). Cuando el personal no se encuentra bien capacitado, es susceptible de cometer ciertos errores que afectan las labores para continuar realizando los proyectos.

1. Se podría generar un daño a la proyección en el sector aeronáutico, debido a errores que impactan en la imagen de la organización; eso no es benéfico.

2. Descuidos por falta de capacitación que podrían generar que un ICE ponga en riesgo la seguridad de las personas en una aeronave y su vida por no haber sido capacitado correctamente en el manejo de ciertos sistemas y equipos de aeronavegación aérea.

3. Aumentar la empatía por la operación mediante nuevos señalamientos, retroalimentación de procedimientos de seguridad, equipos y sistemas donde el ICE podría tener riesgos de algún accidente.

\section{Beneficios al mejorar la curva de aprendizaje}

a) Mejora la productividad si se reduce el tiempo y se perfecciona la mecánica de adaptación del nuevo personal.

b) Atracción y retención de colaboradores, pues los trabajadores están más interesados por un trabajo donde exista un punto de partida.

c) Incrementa la competitividad de las empresas que han aprendido a controlar la rotación y el ausentismo. 
d) Crecimiento del organismo.

e) Genera fidelización de los colaboradores. Al mantener canales de comunicación claros, los procesos definidos se retroalimentan y seguramente se logrará un engagement laboral.

f) Reducción de costos mediante el aumento de la capacitación y la producción.

\section{Competencias sectoriales que predominan en los ICE en SENEAM}

Los ICE encuestados manifestaron, en su mayoría, que el tener una especialización en sus funciones los va a impulsar para desarrollar sus competencias sectoriales, adquirir conocimientos, aumentar la destreza; el contar con cursos de retroalimentación va a favorecerlos con un aprendizaje más rápido y eficiente que los ayudará a tener una menor pérdida de tiempo al realizar sus funciones de trabajo.

Las competencias sectoriales son una base para optimizar la empleabilidad de los ICE, debido al saber obtenido frente a una tarea específica, la cual se hace incuestionable cuando el ICE entra en trato con ella. La competencia va a admitir los conocimientos, saberes y habilidades que nacen de la interacción que se va a efectuar entre el ICE y la tarea.

Las competencias sectoriales son un punto de enfoque integral de formación que va a conectar al mundo laboral y a los ICE con la educación, centrándose en la perfección del capital humano como un principio de innovación, conocimiento, diferenciación y competitividad.

Como lo afirma Miro (2009), las competencias sectoriales se clasifican en competencias transversales o genéricas, competencias técnicas y competencias de sustentabilidad e innovación, las cuales están relacionadas con los conocimientos técnicos que adquieren en su capacitación los ICE con los diferentes equipos y sistemas en el área aeronáutica con los diferentes equipos y sistemas.

Como lo describe la CONFEDI (2016), una competencia es la capacidad de enunciar eficientemente un conjunto de esbozos (arreglos mentales) y valores, admitiendo congregar (poner a disposición) diferentes saberes en un determinado argumento con el fin de resolver contextos profesionales.

Las competencias:

- Manifiestan a capacidades complejas e integradas,

- Están relacionadas con saberes (teóricos, contextuales y procedimentales),

- Se vinculan con el saber hacer (formalizado, empírico, relacional),

- Están referidas al contexto profesional (entendido como la situación en que el profesional debe desempeñarse o ejercer),

- Están referidas al desempeño profesional que se pretende (entendido como la manera en que actúa un profesional técnicamente competente y socialmente comprometido),

- Permiten incorporar la ética y los valores.

El personal técnico aeronáutico capacitado en cursos avalados por la AFAC está habilitado para realizar la intervención de "sistemas electrónicos de tierra y radio ayudas" a la navegación aérea y cuenta con su acreditación de cursos para los sistemas de radar.

Se definieron en el instrumento las siguientes competencias sectoriales de los ICE en SENEAM, las cuales son derivadas de las funciones del grupo unitario 2281 del SINCO (CONOCER, 2017).

\section{Competencias sectoriales} de los ICE en SENEAM

1. Competencia para utilizar de manera efectiva las técnicas y herramientas de ingeniería para resolver fallas técnicas en equipos de comunicaciones aeronáuticas, redes de voz/datos, radionavegación aeronáutica, comunicaciones satelitales, equipos y sistemas de radar.

2. Capacidad para realizar monitoreo, evaluar y ajustar el proceso de funcionamiento en equipos de comunicaciones aeronáuticas, meteorología, redes aeronáuticas, radios ayudas, sistemas satelitales y sistemas de radar.

3. Capacidad de controlar el proceso de instalaciones de equipos de comunicaciones aeronáuticas, 
antenas, redes de voz/datos, equipos de meteorología, sistemas de aeronavegación y sistemas de radar.

4. Capacidad de identificar, formular y resolver fallas de sistemas de comunicaciones, sistemas de meteorología, sistemas de monitoreo remoto.

5. Capacidad de elaborar informes y documentación con especificaciones técnicas derivadas de instalaciones, cambios y servicios generados.

6. Capacidad de incorporar la aplicación de normas oficiales y técnicas referentes a la aeronáutica.

7. Capacidad de controlar las actividades en proceso de instalaciones y demás servicios efectuados.

8. Capacidad de elaborar especificaciones, diagramas, planos para efectuar recomendaciones en los sistemas de comunicación aeronáutica.

9. Capacidad para optimizar el trabajo en equipo en coordinación con personal externo en las diversas actividades requeridas en los servicios.

10. Capacidad para gestionar y controlar procedimientos para la realización de instalaciones, mantenimientos de equipos de comunicación y redes aeronáuticas.

11. Capacidad para realizar la gestión de cotizaciones y recursos financieros para proyectos tecnológicos aeronáuticos.

12. Capacidad para desarrollar informes técnicos referentes a la operación de equipos y sistemas aeronáuticos.

13. Capacidad de desarrollar proyectos identificando las tecnologías disponibles en el mercado.

14. Capacidad de diseñar normas para el control y para el buen servicio de los sistemas.

15. Capacidad para percibir y dirigir actividades de desarrollo de sistemas.

16. Capacidad de realizar una búsqueda para la implementación de sistemas aeronáuticos.

Las competencias sectoriales fueron el resultado de la aplicación de la encuesta en el instrumento y fueron basadas en las funciones a desempeñar por los ICE, que están definidos por el SINCO grupo 2281.

Estas competencias sectoriales se obtienen a través de capacitación. Salgado Benítez (2006) las expone como un aprovechamiento de conocimientos, principalmente de carácter técnico, científico y administrativo. En el desarrollo de los ICE del sector aeronáutico, estas se obtienen a través de los cursos que se les confieren para alcanzar los conocimientos y habilidades necesarias.

El $92 \%$ de los ICE cree que tienen los conocimientos ineludibles para desplegar sus funciones, pero manifiestan la necesidad de retroalimentarse con más conocimientos. Los encuestados exteriorizaron, en su mayoría, que el tener una especialidad en sus funciones los va ayudar para desarrollar sus competencias sectoriales, acrecentar la destreza; asimismo, el contar con un aprendizaje más rápido y eficiente los ayudará a tener una menor pérdida de tiempo al realizar sus funciones de trabajo.

Como lo mencionó Hitt (2008), las habilidades de crecimiento surgen del análisis desde el ambiente interior. Esto significa la utilización de sus propios recursos y capacidades, que será donde se perciban las principales competencias, y donde se van a desarrollar los perfiles competitivos de las funciones de los ingenieros en comunicaciones y electrónica del sector aéreo (Klim, 1993). Chango (2014) señala que las conjeturas de Wright sobre la curva de aprendizaje era que "las horas-hombre necesarias para completar una unidad de producción, decrecerían en un porcentaje constante cada vez que la producción se doblara". Esto implica una mayor producción por parte de un ICE cada vez que su capacitación y su adiestramiento mejoren junto con su experiencia desarrollada, sus habilidades y sus competencias sectoriales, lo que genera un beneficio para el organismo mediante una reducción en los costos de producción - como en su capacitación-al lograrse un mejoramiento de la curva de aprendizaje.

Declaración de conflicto de interés: Los autores no manifiestan conflictos de interés institucionales ni personales.

\section{Referencias}

Aca, N. (2017). Cómo reducir la curva de aprendizaje de tus colaboradores. Merca2.0. 
Arrow, K. J. (1962). The Economic Implications of Learning by Doing. EUA.

Ballesteros P., Ballesteros, D., \& Jaramillo, C. (2005). Aplicación de la lúdica en la curva de aprendizaje. Scientia Et Technica, 1(27).

Chango, Z. (2014). Las Curvas de Aprendizaje. Universidad de las Fuerzas Armadas Ecuador.

CONFEDI. (2016). Competencias y perfil del ingeniero iberoamericano, formación de profesores y desarrollo tecnológico e innovación. ASIBEI.

CONOCER. (2017). Competencias de personas y perfiles ocupacionales. https://conocer.gob.mx/wp-content/up loads/2017/05/MonografiasPerfilesOcupacionalesTo tales.pdf

Dessler, G., \& Varela, R. (2011). Administración de Recursos Humanos. Pearson Prentice Hall.

DOF. (1978). Diario Oficial de la Federación. DOF. http://www. dof.gob.mx/copias.php?acc=ajaxPaginas\&paginas= 1-25\&seccion=SEGUNDA\&edicion=199171\&ed=MATUTI NO\&fecha $=25 / 11 / 1950$

Hirschmann, W. B. (1964). Beneficio de la curva de aprendizaje. Harvard Business Review.

Hitt, I. (2008). Administración estratégica. CENGAGE Learning.

Hyrkäs, K., Appelqvist-Schmidlechner, K., \& Oksa, L. (2003). Validating an Instrument for Clinical Supervision Using an Expert Panel. International Journal of Nursing Studies, 40(6), 619-625.

INEGI. (2011). Sistema nacional de clasificación de ocupaciones 2011 SINCO. INEGI.

Jacobs, R. B. (2014). Administracion de operaciones, producción y cadena de suministros. McGraw-Hill.

Kanawaty, G. (1995). Introducción al estudio del trabajo. Oficina Internacional del Trabajo Ginebra.

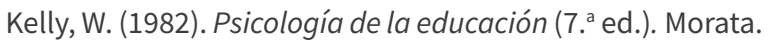

Kim, D. (1993). The Link between Individual and Organizational Learning. MIT Solan.

Krajenski, L. Y. (2000). Administración de operaciones, estrategia y análisis (5. ${ }^{\mathrm{a}} \mathrm{ed}$.). Pearson.
Lefcovich, M. (2003). Kaizen. Mejora continua y cuadro de mando integral. Gestiopolis. https://www.gestiopolis.com/ kaizen-mejora-continua-y-cuadro-de-mando-integral/

Martínez Gutiérrez, R. (2012). Quinta Hélice Sistémica (qhs), un modelo para el desarrollo de políticas públicas. Cooperativismo \& Desarrollo, 20(101). https://revistas.ucc.edu. co/index.php/co/article/view/17

Martínez-Gutierrez, R. (2020). Methodology of Dictionaries of Sector Competences (DCS), to Design Standards of Professional Competences, Research and Labor. In International Conference on Applied Human Factors and Ergonomics (pp. 323-329). Springer.

Miro, J. (2009, 11 de marzo). Dimecres. www.fib.upc.edu.

Rojas, V. M. (2011). Metodología de la investigación (Diseño y ejecución). Ediciones de la U.

Salgado Benítez, J. (2006). Administración de Recursos Huma$\operatorname{nos}\left(2^{\mathrm{a}}\right.$ ed.). Grupo Éxodo.

Sampieri, R. (2010). Metodología de la investigación (4a ed.). McGraw Hill.

Skjong, R., \& Wentworth, B. (2000). Expert Judgement and Risk Perception. http://research.dnv.com/skj/Papers/Skj Wen.pdf

Terrazas, L. A., Aldape, A., \& Tarango, L. (2009). La curva de aprendizaje como estrategia para reducción de costos. $2^{\text {do }}$ Congreso Internacional de Investigación, Chihuahua, México.

Titone, R. (1986). Psicodidáctica (4. ${ }^{\mathrm{a}}$ ed.). Narcea Ediciones.

Willard, I., \& Kantor, P. (1998). Toward a Theory of Continuous Improvement and the Learning Curve. Management Science, 44(7), 910-920. https://www.researchgate.net/ publication/227447135_Toward_a_Theory_of_Continuous_ Improvement_and_the_Learning_Curve

Wright, T. P. (1936). Factors Affecting the Cost of Airplanes. Journal of the Aeronautical Sciences, 3, 122-128.

Yelle, L. E. (1979). La curva de aprendizaje: revisión histórica y encuesta completa. Wiley Online Library. 